\title{
Bei akuter Appendizitis schnell in den OP
}

\author{
Kinder mit akuter Appendizitis brauchen hellwache Ärzte und eine gut organisierte Klinik: \\ Mit jeder Stunde Verzögerung auf dem Weg in den OP steigt die Perforationsgefahr um $10 \%$.
}

— Die akute Appendizitis ist die häufigste chirurgische Notfallsituation bei Kindern. Ist die Diagnose erst einmal gestellt, beginnt ein Wettlauf mit der Zeit, da die Perforation der Appendix die Operationsbedingungen massiv verschlechtert und mit einer erhöhten Morbidität und Mortalität einhergeht. Das bedeutet, dass Verdachtsfälle schnell abgeklärt werden müssen und Krankenhäuser optimal organisiert sein müssen, um Verzögerungen bis zur Operation zu vermeiden.

Wie wichtig dies ist, wurde nun in einer Untersuchung aus einem New Yorker Krankenhaus gezeigt. Man analysierte aus einer vierjährigen Beobachtungsperiode retrospektiv 248 Fälle von Kindern mit CT-bestätigter unkomplizierter Appendizitis. Alle Kinder wurden schließlich appendektomiert. Obwohl alle bereits in der Nothilfe parenteral mit Antibiotika behandelt wurden, entwickelten 54 Kinder im Krankenhaus eine Perforation der Appendix. Bei 52 von ihnen $(96,4 \%)$ dauerte es nach der Erstvorstellung in der Nothilfe länger als neun Stunden bis zur Operation.

Trennt man die Kinder anhand der Wartezeit in zwei Gruppen, so ergibt sich eine Perforationsrate von $4,6 \%$ für jene, die binnen neun Stunden operiert wurden. Für die länger Wartenden war die Rate sechsmal höher (25\%). Von den Kindern, die $\geq 24$ Stunden warten mussten, waren sogar $41 \%$ betroffen.

In einer Regressionsanalyse erwiesen sich drei Faktoren als signifikant assoziiert mit einer Perforation: die Zeitdauer zwischen der Vorstellung in der Nothilfe und dem Eintreffen im OP, das Vorliegen von Fieber und das Vorhandensein von Appendixsteinen. Für jede Stunde Verzögerung bis zur Operation erhöhte sich das Risiko für eine Perforation der Appendix um 10\%.
- Bonadio Wet al. Impact of in-hospital timing to appendec tomy on perforation rates in children with appencitis. JEmergency Medicine. 2015;49:597-604

\section{KOMMENTAR}

Die Untersuchung mutet für deutsche Verhältnisse etwas merkwürdig an. Ein Kind mit akuter Appendizitis wird hierzulande operiert, sobald die Diagnose gestellt ist. Das Problem besteht bei uns eher in der Diagnosestellung. Eine von den Autoren zitierte Umfrage unter 500 US-amerikanischen Kinderchirurgen ergab dagegen ein ganz anderes Bild. $96 \%$ von ihnen sahen eine nicht perforierte Appendizitis nicht als chirurgischen Notfall an. 92\% glaubten, ein Aufschieben der Appendektomie über die Nacht hinweg bis zum nächsten Morgen würde nicht mit einer wesentlichen Erhöhung des Perforationsrisikos einhergehen. Zwei Drittel gaben $z u$, dass an ihrer Abteilung keine Verfahrensanweisung für derartige Situationen bestehe. Glückliches Deutschland!

Prof. Dr. med. H. S. Füeß।

\section{Ein Gerstenkorn ist das jedenfalls nicht!}

Am rechten Oberlid einer 82-jährigen Frau war es in den vergangenen drei Monaten zu einer rasch zunehmenden Rötung, einer Schwellung und schließlich einer Ulzeration gekommen. Der Hausarzt hatte anfangs, als der Befund

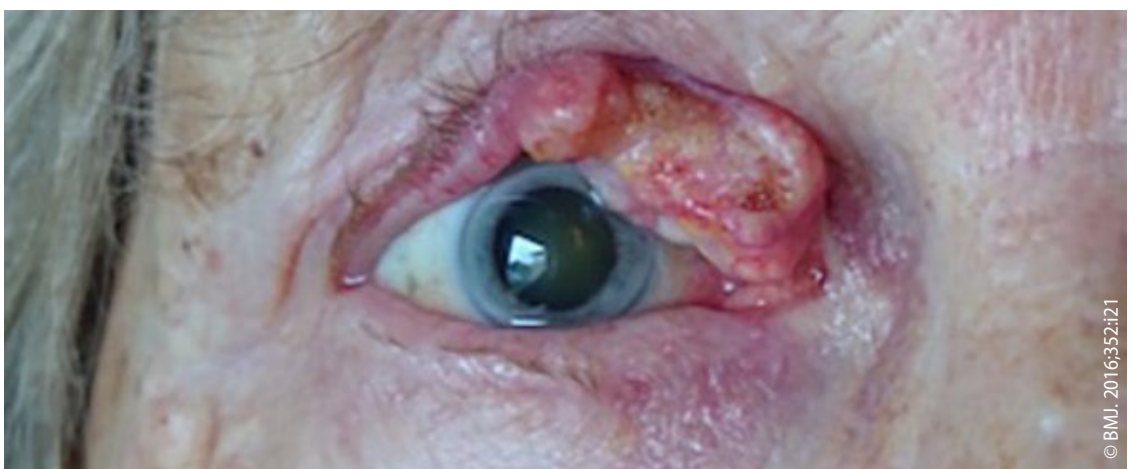

Große, zentral exulzerierte Raumforderung am rechten Augenlid. noch weniger ausgeprägt war, an ein Gerstenkorn gedacht und mit antibiotischer Salbe behandelt. Der Patientin selbst war das rasche Größenwachstum der Läsion zunächst nicht aufgefallen. Ein Verwandter machte sie schließ-

lich darauf aufmerksam und brachte sie erneut zum Hausarzt, der sie eilig an eine Augenklinik überwies.

Bei Aufnahme zeigte sich eine große, zentral exulzerierte Raumforderung, die fast das gesamte rechte Oberlid einnahm und zur weitgehenden Zerstörung des Oberlidgewebes geführt hatte. Die Histologie ergab ein lokalinvasiv wachsendes Plattenepithelkarzinom. Plattenepithelkarzinome kommen an den Lidern zwar 40-mal seltener vor als Basaliome, sie sind aber wesentlich aggressiver, zerstören rasch die umgebenden Strukturen und erhöhen das Risiko einer lymphatischen regionalen Metastasierung.

Junge Frauen schauen vielleicht zu oft in den Spiegel, ältere bisweilen aber zu selten.

Prof. Dr. med. H. S. FüeßI

- Hassan A et al. (alishassan@doctors.org.uk) Rapidlyenlarging eyelid lesion. BMJ. 2016;352:i21 